\title{
Uniquely Complemented Posets
}

\author{
Ivan Chajda ${ }^{1} \cdot$ Helmut Länger ${ }^{2} \cdot$ Jan Paseka $^{3}$
}

Received: 14 November 2016 / Accepted: 17 October 2017 / Published online: 6 November 2017

(C) The Author(s) 2017. This article is an open access publication

\begin{abstract}
We study complementation in bounded posets. It is known and easy to see that every complemented distributive poset is uniquely complemented. The converse statement is not valid, even for lattices. In the present paper we provide conditions that force a uniquely complemented poset to be distributive. For atomistic resp. atomic posets as well as for posets satisfying the descending chain condition we find sufficient conditions in the form of so-called LU-identities. It turns out that for finite posets these conditions are necessary and sufficient.
\end{abstract}

Keywords Complemented poset $\cdot$ Uniquely complemented · Atomic · Atomistic · Distributive · LU-identity

Support of the research by the Austrian Science Fund (FWF), project I 1923-N25, and the Czech Science Foundation (GACR), project 15-34697L, as well as of the research of the third author by the Czech Science Foundation (GAČR), project 15-15286S, is gratefully acknowledged.

\section{Ivan Chajda}

ivan.chajda@upol.cz

Helmut Länger

helmut.laenger@tuwien.ac.at

Jan Paseka

paseka@math.muni.cz

1 Faculty of Science, Department of Algebra and Geometry, Palacký University Olomouc, 17. listopadu 12, 77146 Olomouc, Czech Republic

2 Faculty of Mathematics and Geoinformation, Institute of Discrete Mathematics and Geometry, Wiedner Hauptstraße 8-10, 1040, Vienna, Austria

3 Faculty of Science, Department of Mathematics and Statistics, Masaryk University Brno, Kotlářská 2, 61137 Brno, Czech Republic 


\section{Introduction}

It is well-known and a fairly elementary result that every complemented distributive lattice is uniquely complemented and hence Boolean. For finite or even atomic lattices the converse assertion is valid, i.e. every atomic uniquely complemented lattice is distributive. However, in 1945 R. P. Dilworth ([6]) disproved the conjecture that every uniquely complemented lattice is distributive by showing that every lattice is, indeed, a sublattice of a uniquely complemented lattice. In other words, no lattice property which is preserved under the formation of sublattices will be valid in all uniquely complemented lattices. In particular, there exists a uniquely complemented lattice containing $N_{5}$ as a sublattice and hence it must be non-modular. A construction of such a lattice was presented by V. N. Salii ([14]). A number of conditions forcing a uniquely complemented lattice to be distributive was formulated by several authors. One of the most profound is the BirkhoffWard Theorem ([1, 7]). A necessary and sufficient condition formulated in the form of an identity in two variables was presented in [4], for several other such identities see e.g. [3].

The natural question arises if the aforementioned results and methods can be transferred also to posets. An important progress in this direction was already obtained by B. N. Waphare and V. V. Joshi in [15]. They formulated such conditions by means of pseudocomplements and so-called regular elements.

Our approach is different. We are going to introduce simple LU-identities in two variables which are satisfied in every complemented distributive poset such that every uniquely complemented poset satisfying one of these LU-identities must be distributive. Several other results concerning uniquely complemented posets are obtained in the case of atomicity.

\section{Basic Concepts}

In this section we summarize all concepts used throughout the paper and also some results which can be found in the references. All posets considered in this paper are assumed to be bounded having 0 as least and 1 as greatest element. Let $\mathbf{P}=(P, \leq, 0,1)$ be a bounded poset. For each subset $A$ of $P$ let

$$
U(A):=\{y \in P \mid x \leq y \text { for all } x \in A\}
$$

denote the upper cone of $A$ and

$$
L(A):=\{y \in P \mid y \leq x \text { for all } x \in A\}
$$

the lower cone of $A$. We write $U(a, A)$ instead of $U(\{a\} \cup A), U(a)$ instead of $U(\{a\}), L(a)$ instead of $L(\{a\})$ and so on. Then we have

- $A \subseteq B$ implies $U(B) \subseteq U(A)$ and $L(B) \subseteq L(A)$,

- $U(L(U(A)))=U(A)$ and $L(U(L(A)))=L(A)$,

- $U(L(a))=U(a)$ and $L(U(a))=L(a)$,

- $U(a, A)=U(L(a), A)$ and $L(a, A)=L(U(a), A)$,

- $U(A \cup B)=U(A) \cap U(B)$ and $L(A \cup B)=L(A) \cap L(B)$,

- the following are equivalent: $a \leq b ; U(a) \supseteq U(b) ; L(a) \subseteq L(b)$,

- the following are equivalent: $a=b ; U(a)=U(b) ; L(a)=L(b)$.

If $\mathbf{P}$ is a lattice $(P, \vee, \wedge)$ then $U(a, b)=U(a \vee b)$ and $L(a, b)=L(a \wedge b)$. 
Lemma 2.1 In every poset the following conditions are equivalent:

(i) $U(L(x, y), z) \approx U(L(U(x, z), U(y, z)))$,

(ii) $L(U(L(x, y), z)) \approx L(U(x, z), U(y, z))$,

(iii) $L(U(x, y), z) \approx L(U(L(x, z), L(y, z)))$,

(iv) $U(L(U(x, y), z)) \approx U(L(x, z), L(y, z))$.

Proof Obviously, (i) and (ii) are equivalent and so are (iii) and (iv). The equivalence of (i) and (iii) was shown in [5]. For the reader's convenience we provide a proof. We show that (i) implies (iii). The converse implication follows by duality. Hence assume (i). Then

$$
\begin{aligned}
L(U(L(x, z), L(y, z))) & \approx L(U(L(U(x, L(y, z)), U(z, L(y, z))))) \\
& \approx L(U(x, L(y, z)), U(z, L(y, z))) \approx L(U(x, L(y, z)), U(z)) \\
& \approx L(U(L(y, z), x), U(z)) \approx L(U(L(U(y, x), U(z, x))), U(z)) \\
& \approx L(U(L(U(x, y), U(x, z))) \cup U(z)) \\
& \approx L(U(L(U(x, y), U(x, z)))) \cap L(U(z)) \\
& \approx L(U(x, y) \cup U(x, z)) \cap L(U(z)) \\
& \approx L((U(x, y) \cup U(x, z)) \cup U(z)) \\
& \approx L(U(x, y) \cup(U(x, z) \cup U(z)) \approx L(U(x, y) \cup U(z)) \\
& \approx L(U(x, y), U(z)) \approx L(U(x, y), z)
\end{aligned}
$$

Recall from [10] or [13] that a poset $(P, \leq)$ is called distributive if it satisfies any of the equivalent conditions (i) - (iv) of Lemma 2.1. Distributive posets were investigated in [5].

In what follows, expressions like (i) - (iv) of Lemma 2.1 will be called LU-identities. They replace in a certain sense lattice identities for posets that are not lattices. In particular, every poset satisfies the following LU-identities:

$$
\begin{array}{ll}
U(x, x)=U(x), & L(x, x)=L(x) \\
U(x, y)=U(y, x), & L(x, y)=L(y, x) \\
U(U(x, y), z)=U(x, U(y, z)), & L(L(x, y), z)=L(x, L(y, z)) \\
U(x, L(x, y))=U(x), & L(x, U(x, y))=L(x)
\end{array}
$$

which can be considered as a generalization of the identities defining lattices.

Moreover, every poset obviously satisfies the following inclusions:

$$
\begin{aligned}
U(L(x, y), z) & \supseteq U(L(U(x, z), U(y, z))), \\
L(U(L(x, y), z)) & \subseteq L(U(x, z), U(y, z)), \\
L(U(x, y), z) & \supseteq L(U(L(x, z), L(y, z))) \text { and } \\
U(L(U(x, y), z)) & \subseteq U(L(x, z), L(y, z)) .
\end{aligned}
$$

Hence for proving distributivity of a poset it suffices to prove the converse inclusion of one of the above inclusions.

The connection between distributive posets and distributive lattices is described by the following lemma:

Lemma 2.2 A lattice is a distributive poset if and only if it is a distributive lattice. 
Proof Let $\mathbf{L}=(L, \vee, \wedge)$ be a lattice. Then $\mathbf{L}$ is distributive if and only if it satisfies the identity $(x \vee y) \wedge z \approx(x \wedge z) \vee(y \wedge z)$ which is equivalent to the LU-identity

$$
U((x \vee y) \wedge z) \approx U((x \wedge z) \vee(y \wedge z)) .
$$

Now we have

$U((x \vee y) \wedge z) \approx U(L((x \vee y) \wedge z)) \approx U(L(x \vee y, z)) \approx U(L(U(x \vee y), z)) \approx U(L(U(x, y), z))$ and

$U((x \wedge z) \vee(y \wedge z)) \approx U(x \wedge z, y \wedge z) \approx U(L(x \wedge z), L(y \wedge z)) \approx U(L(x, z), L(y, z))$.

Hence LU-identity (1) is equivalent to (iv) of Lemma 2.1.

The following concept was introduced in [5]: A poset $(P, \leq)$ is called modular if it satisfies the implication

$$
x \leq z \Rightarrow L(U(x, y), z)=L(U(x, L(y, z)))
$$

$(x, y, z \in P)$. It is easy to check that every distributive poset is modular.

Let $\mathbf{P}=(P, \leq, 0,1)$ be a bounded poset and $a, b \in P$. $\mathbf{P}$ is said to be a De Morgan poset if it is equipped with a unary operation ' on $P$ such that $\left(x^{\prime}\right)^{\prime}=x$ and such that $x \leq y$ implies $y^{\prime} \leq x^{\prime}$ for all $x, y \in P$. Recall from [2] that $b$ is called a complement of $a$ if $U(a, b)=\{1\}$ and $L(a, b)=\{0\}$. The poset $\mathbf{P}$ is called complemented if every element of $P$ has at least one complement and uniquely complemented if every element of $P$ has exactly one complement. A unary operation ' on $P$ is called a complementation of $\mathbf{P}$ if $x^{\prime}$ is a complement of $x$ for all $x \in P$. A complementation ' of $\mathbf{P}$ is called an orthocomplementation of $\mathbf{P}$ if $\mathbf{P}$ is a De Morgan poset. In such a case, $\mathbf{P}$ will be called an orthocomplemented poset. If $\mathbf{P}$ is uniquely complemented then the mapping ' assigning to each element of $P$ its complement is in fact a complementation and, moreover $\left(x^{\prime}\right)^{\prime}=x$ for every $x \in P$. An element $b$ is called the pseudocomplement of $a$ if $L(a, b)=\{0\}$ and if $x \leq b$ for each $x \in P$ with $L(a, x)=\{0\}$. The poset $\mathbf{P}$ is called pseudocomplemented if each element of $P$ has a pseudocomplement.

Proposition 2.3 ([2]) Every element of a distributive poset has at most one complement.

Proposition 2.4 ([11]) Every uniquely complemented pseudocomplemented poset is distributive.

In accordance with Propositions 2.3 and 2.4 we present the following examples.

Example 2.5 The poset given by the Hasse diagram

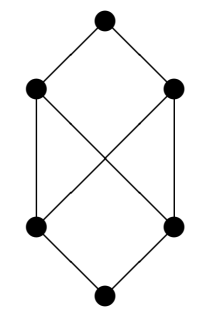

is distributive and pseudocomplemented, but neither a lattice nor complemented. 
Now we present some examples of uniquely complemented posets.

Example 2.6 The posets given by the Hasse diagrams
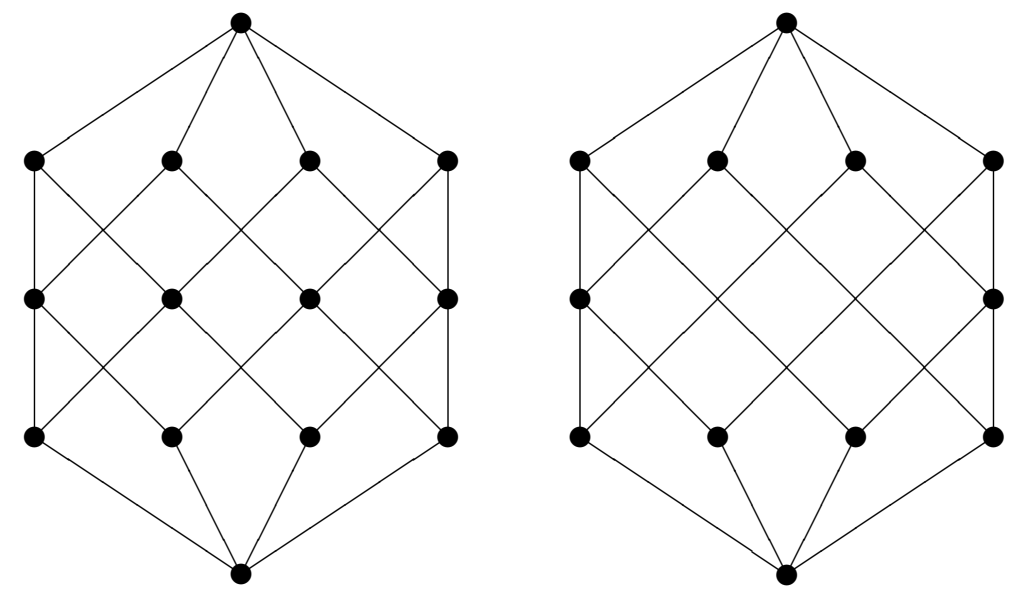

are distributive and uniquely complemented, but not lattices.

Uniquely complemented posets were treated by B. N. Waphare and V. V. Joshi ([15]). We will use different tools and methods and state other conditions formulated in a different language. In the following we need some more concepts.

Let $\mathbf{P}=(P, \leq, 0,1)$ be a bounded poset. An element of $P$ is called an atom of $\mathbf{P}$ if it covers 0 and it is called a dual atom of $\mathbf{P}$ if it is covered by 1 . The poset $\mathbf{P}$ is called atomic if for each $a \in P \backslash\{0\}$ there exists an atom $p$ of $\mathbf{P}$ with $p \leq a$ and it is called atomistic if every of its elements is a supremum of atoms. Of course, every atomistic or finite bounded poset is atomic. On the other hand, a finite atomic bounded poset need not be atomistic.

For a bounded poset $\mathbf{P}=\left(P, \leq,^{\prime}, 0,1\right)$ with unary operation and a subset $A$ of $P$ put $A^{\prime}:=\left\{x^{\prime} \mid x \in A\right\}$.

A poset $\mathbf{P}=\left(P, \leq,^{\prime}, 0,1\right)$ with complementation is said to satisfy the De Morgan laws if it satisfies the LU-identities

$$
(U(x, y))^{\prime} \approx L\left(x^{\prime}, y^{\prime}\right) \text { and }(L(x, y))^{\prime} \approx U\left(x^{\prime}, y^{\prime}\right) .
$$

The following result was proved in [2]:

Proposition 2.7 A uniquely complemented poset $\mathbf{P}=\left(P, \leq,^{\prime}, 0,1\right)$ satisfies the De Morgan laws if and only if $x \leq y$ implies $y^{\prime} \leq x^{\prime}(x, y \in P)$.

It should be mentioned that in a poset with complementation satisfying the De Morgan laws every LU-identity is equivalent to its dual obtained by interchanging $L$ and $U$ as well as $x$ and $x^{\prime}$.

\section{Two LU-identities}

According to Proposition 2.3, every complemented distributive poset is uniquely complemented. It is evident that the converse does not hold, i.e. there exist uniquely complemented 
posets (even lattices, see [6] or [14]) which are not distributive. On the other hand, every uniquely complemented atomic lattice (in particular, every finite lattice) is distributive, see the so-called Birkhoff-Ward Theorem $([1,7])$. Hence the question arises if a similar result holds also for uniquely complemented posets.

For bounded posets $\mathbf{P}=\left(P, \leq,{ }^{\prime}, 0,1\right)$ with a unary operation ' we introduce the following two LU-identities:

$$
\begin{aligned}
& U\left(x^{\prime}, L(x, y)\right) \approx U\left(x^{\prime}, y\right), \\
& L\left(x^{\prime}, U(x, y)\right) \approx L\left(x^{\prime}, y\right) .
\end{aligned}
$$

It is evident that for De Morgan posets these LU-identities are equivalent.

Now we can prove the following:

Theorem 3.1 Every complemented distributive poset satisfies both LU-identities (2) and (3).

Proof In a complemented distributive poset we have

$$
\begin{aligned}
U\left(x^{\prime}, L(x, y)\right) & \approx U\left(L(x, y), x^{\prime}\right) \approx U\left(L\left(U\left(x, x^{\prime}\right), U\left(y, x^{\prime}\right)\right)\right) \approx U\left(L\left(1, U\left(y, x^{\prime}\right)\right)\right) \\
& \approx U\left(L\left(U\left(y, x^{\prime}\right)\right)\right) \approx U\left(y, x^{\prime}\right) \approx U\left(x^{\prime}, y\right)
\end{aligned}
$$

and

$$
\begin{aligned}
L\left(x^{\prime}, U(x, y)\right) & \approx L\left(U(x, y), x^{\prime}\right) \approx L\left(U\left(L\left(x, x^{\prime}\right), L\left(y, x^{\prime}\right)\right)\right) \approx L\left(U\left(0, L\left(y, x^{\prime}\right)\right)\right) \\
& \approx L\left(U\left(L\left(y, x^{\prime}\right)\right)\right) \approx L\left(y, x^{\prime}\right) \approx L\left(x^{\prime}, y\right) .
\end{aligned}
$$

Now we are going to show that, conversely, bounded posets with a unary operation satisfying both LU-identities (2) and (3) have some interesting properties.

Lemma 3.2 For a complemented poset $\mathbf{P}=\left(P, \leq,{ }^{\prime}, 0,1\right)$ the following hold:

(i) If $\mathbf{P}$ satisfies $L U$-identity (2) then it is pseudocomplemented.

(ii) If $\mathbf{P}$ satisfies one of the LU-identities (2) and (3) then' is antitone, i.e. $x \leq y$ implies $y^{\prime} \leq x^{\prime}(x, y \in P)$.

Proof Let $a, b \in P$.

(i) We have $L\left(a, a^{\prime}\right)=\{0\}$ and if $L(a, b)=\{0\}$ then

$$
a^{\prime} \in U\left(a^{\prime}\right)=U\left(a^{\prime}, 0\right)=U\left(a^{\prime}, L(a, b)\right)=U\left(a^{\prime}, b\right) \subseteq U(b),
$$

i.e. $b \leq a^{\prime}$.

(ii) Assume $a \leq b$. Then $L\left(b, b^{\prime}\right)=\{0\}$ and $U\left(a, a^{\prime}\right)=\{1\}$ and hence $L\left(a, b^{\prime}\right)=\{0\}$ and $U\left(b, a^{\prime}\right)=\{1\}$. Now LU-identity (2) implies

$$
a^{\prime} \in U\left(a^{\prime}\right)=U\left(a^{\prime}, 0\right)=U\left(a^{\prime}, L\left(a, b^{\prime}\right)\right)=U\left(a^{\prime}, b^{\prime}\right) \subseteq U\left(b^{\prime}\right)
$$

whereas LU-identity (3) implies

$$
b^{\prime} \in L\left(b^{\prime}\right)=L\left(b^{\prime}, 1\right)=L\left(b^{\prime}, U\left(b, a^{\prime}\right)\right)=L\left(b^{\prime}, a^{\prime}\right) \subseteq L\left(a^{\prime}\right),
$$

i.e. $b^{\prime} \leq a^{\prime}$ in both cases.

In the following lemma we do not assume that the unary operation ' on $P$ is a complementation. Nevertheless, we show that if $\mathbf{P}$ satisfies both LU-identities (2) and (3) then ' is not only a complementation of $\mathbf{P}$, but $\mathbf{P}$ is even uniquely complemented. This demonstrates the crucial role of these LU-identities. 
Lemma 3.3 Every bounded poset $\mathbf{P}=\left(P, \leq,{ }^{\prime}, 0,1\right)$ with a unary operation satisfying both LU-identities (2) and (3) is uniquely complemented and hence' is an involution, i.e. the identity $\left(x^{\prime}\right)^{\prime} \approx x$ holds.

Proof Let $a, b \in P$. Then

$$
U\left(x, x^{\prime}\right) \approx U\left(x^{\prime}, x\right) \approx U\left(x^{\prime}, L(x)\right) \approx U\left(x^{\prime}, L(x, 1)\right) \approx U\left(x^{\prime}, 1\right) \approx\{1\}
$$

and

$$
L\left(x, x^{\prime}\right) \approx L\left(x^{\prime}, x\right) \approx L\left(x^{\prime}, U(x)\right) \approx L\left(x^{\prime}, U(x, 0)\right) \approx L\left(x^{\prime}, 0\right) \approx\{0\} .
$$

Hence' is a complementation. Now assume $U(a, b)=\{1\}$ and $L(a, b)=\{0\}$. Then

$$
a^{\prime} \in U\left(a^{\prime}\right)=U\left(a^{\prime}, 0\right)=U\left(a^{\prime}, L(a, b)\right)=U\left(a^{\prime}, b\right) \subseteq U(b)
$$

and hence $b \leq a^{\prime}$. Moreover,

$$
a^{\prime} \in L\left(a^{\prime}\right)=L\left(a^{\prime}, 1\right)=L\left(a^{\prime}, U(a, b)\right)=L\left(a^{\prime}, b\right) \subseteq L(b)
$$

and hence $a^{\prime} \leq b$. This shows $b=a^{\prime}$ and hence $\mathbf{P}$ is uniquely complemented. Since $a$ is a complement of $a^{\prime}$ we obtain $\left(a^{\prime}\right)^{\prime}=a$.

Lemma 3.4 For a uniquely complemented poset LU-identities (2) and (3) are equivalent.

Proof First observe $\left(x^{\prime}\right)^{\prime} \approx x$. If LU-identity (2) holds and $z \in L\left(x^{\prime}, U(x, y)\right)$ then ' is antitone according to Lemma 3.2 and hence it is a De Morgan poset, i.e. LU-identities (2) and (3) are equivalent.

Summarizing the previous lemmata we obtain

Theorem 3.5 Every uniquely complemented poset $\mathbf{P}=\left(P, \leq,{ }^{\prime}, 0,1\right)$ satisfying $L U$ identity (2) is distributive.

Proof Lemma 3.2 and Proposition 2.4.

Corollary 3.6 Every bounded poset $\mathbf{P}=\left(P, \leq,{ }^{\prime}, 0,1\right)$ with a unary operation satisfying both LU-identities (2) and (3) is uniquely complemented, orthocomplemented and distributive.

Proof Lemma 3.3, Lemma 3.2 and Theorem 3.5.

Now we are able to present a solution of the problem mentioned at the beginning.

\section{Corollary 3.7}

(i) A complemented poset is distributive if and only if it satisfies both LU-identities (2) and (3).

(ii) A uniquely complemented poset is distributive if and only if it satisfies LU-identity (2).

Proof

(i) Theorem 3.1, Lemma 3.3 and Theorem 3.5.

(ii) Theorem 3.1 and Theorem 3.5. 


\section{Distributivity of Uniquely Complemented Posets}

As shown above, every distributive complemented poset is uniquely complemented. In this section we provide conditions forcing a uniquely complemented poset to be distributive. It is not surprising that atomicity will play an essential role in this context because of the role this property plays within uniquely complemented lattices. We start with two preliminary results.

Lemma 4.1 If $\mathbf{P}=\left(P, \leq,{ }^{\prime}, 0,1\right)$ is a uniquely complemented atomic poset and A denotes the set of its atoms then the following hold:

(O1) If $a, b \in P$ and $a>b$ then there exists an atom $p$ of $\mathbf{P}$ with $p \leq a$ and $p \wedge b=0$.

(O2) The complement of an element of $A$ is a coatom of $\mathbf{P}$.

(O3) If $p, q \in A$ and $p \neq q$ then $q \leq p^{\prime}$.

Proof (O1) Evidently, $a>b$ yields $b^{\prime} \vee a=1$. We therefore cannot have $b^{\prime} \wedge a=0$ by the uniqueness of complements. It follows that there exists an atom $p$ of $\mathbf{P}$ with $p \leq b^{\prime}$ and $p \leq a$. Since $p \leq b^{\prime}$ we have $p \wedge b=0$.

(O2) Let $p \in A$. Then $p^{\prime} \neq 1$ since otherwise we would have the contradiction $p=0$. Suppose that $p^{\prime} \leq a<1$ for some $a \in P$. Then $p \vee a=1$. Now $p \leq a$ would imply $a=p \vee a=1$, a contradiction. Hence $p \not \leq a$ whence $p \wedge a=0$ and consequently $a=p^{\prime}$ by the uniqueness of complements. Hence $p^{\prime}$ is a coatom of $\mathbf{P}$.

(O3) By condition (O2), both $p^{\prime}$ and $q^{\prime}$ are coatoms of $\mathbf{P}$. Now $q \not \leq p^{\prime}$ would imply $q \vee p^{\prime}=1$ and $q \wedge p^{\prime}=0$, i.e. $p=q$, a contradiction.

In the next proposition we show when a uniquely complemented atomic poset $\mathbf{P}$ satisfying a particular condition can be embedded into the Boolean algebra $2^{A}$ where $A$ denotes the set of all atoms of $\mathbf{P}$.

Proposition 4.2 If $\mathbf{P}=\left(P, \leq,^{\prime}, 0,1\right)$ is a uniquely complemented atomic poset, A denotes the set of its atoms and the following condition holds:

(O4) If $p \in A$ and $a \in P$ then $p \wedge a=0$ if and only if $a \leq p^{\prime}$.

then $f: P \rightarrow 2^{A}$ defined by $f(x):=\{p \in A \mid p \leq x\}$ for all $x \in P$ is an injective mapping of bounded posets preserving complementation as well as existing joins and meets.

Proof Let $a, b \in P$ and $p \in A$. Note first that $a \leq b$ implies $f(a) \subseteq f(b)$ and that $f(0)=\emptyset$ and $f(1)=A$. We establish the proof by means of the following sequence of observations:

(O5) If $p \in A$ and $S$ is a non-empty subset of $P$ then $p \in L(U(S))$ if and only if $p \leq s$ for some $s \in S$.

Suppose that $p \leq s$ for some $s \in S$. Then $p \in L(U(s)) \subseteq L(U(S))$. Conversely, let $p \in L(U(S))$. For each $s \in S$ we have either $p \leq s$ or $p \not \leq s$, i.e., either $p \leq s$ or, by condition (O4), $s \leq p^{\prime}$. Now we cannot have $s \leq p^{\prime}$ for all $s \in S$ since then $p^{\prime} \in U(S)$, which gives $p \leq p^{\prime}$, i.e., $p=p \wedge p^{\prime}=0$, a contradiction. Hence there exists some $s \in S$ with $p \leq s$.

Now assume that $S$ is a non-empty subset of $P$ and $\bigvee S$ exists. From condition (O5) we obtain that $p \leq \bigvee S$ if and only if $p \leq s$ for some $s \in S$, i.e., $f(\bigvee S)=\bigcup\{f(s) \mid s \in S\}$. If $\bigwedge S$ exists then, obviously, $f(\bigwedge S)=\bigcap\{f(s) \mid s \in S\}$. Moreover, for all $x \in P$, $f(0)=\emptyset=f(x) \cap f\left(x^{\prime}\right)$ and, according to condition (O5), $f(1)=A=f(x) \cup f\left(x^{\prime}\right)$. 
This yields $f\left(x^{\prime}\right)=A \backslash(f(x))$ for all $x \in P$. Hence $f$ is a morphism of bounded posets preserving complementation and arbitrary existing joins and meets.

(O6) If $a \in P$ then $\bigvee(f(a))=1$ if and only if $a=1$.

Assume first $\bigvee(f(1)) \neq 1$. Then there exists some $b \in(U(A)) \backslash\{1\}$. Now we have $f\left(b^{\prime}\right)=A \backslash(f(b))=A \backslash A=\emptyset$ and hence $b^{\prime}=0$, i.e. $b=1$, a contradiction. Hence $\bigvee(f(1))=1$. Now assume $a \neq 1$. Then $a^{\prime} \neq 0$. Hence there exists an atom $p \in \mathbf{P}$ with $p \leq a^{\prime}$ whence $p \wedge a=0$ which implies $a \leq p^{\prime}$ according to condition (O4). Now $p^{\prime} \in(U(f(a))) \backslash\{1\}$ and hence $\bigvee(f(a)) \neq 1$.

(O7) $f$ is injective.

Suppose that $f(a)=f(b)$. Let us check that $b$ is a complement of $a^{\prime}$. We have from conditions (O6) and (O5) that $1=\bigvee A=\bigvee\left(f(a) \cup f\left(a^{\prime}\right)\right)=\bigvee\left(f(b) \cup f\left(a^{\prime}\right)\right)$ and hence $U\left(b, a^{\prime}\right)=\{1\}$. Assume $L\left(b, a^{\prime}\right) \neq\{0\}$. Then there exists an element $c$ of $P \backslash\{0\}$ with $c \leq b$ and $c \leq a^{\prime}$. Since $\mathbf{P}$ is atomic there exists an atom $p$ of $\mathbf{P}$ with $p \leq c$. Now we have $p \in f(b) \cap f\left(a^{\prime}\right)=f(a) \cap f\left(a^{\prime}\right)=\emptyset$, a contradiction. Hence $L\left(b, a^{\prime}\right)=\{0\}$ and $b$ is a complement of $a^{\prime}$. But $\mathbf{P}$ is uniquely complemented and therefore $a=b$.

Although it was shown that every uniquely complemented atomic poset $\mathbf{P}$ satisfying condition (O4) can be embedded into a distributive complemented lattice, $\mathbf{P}$ need not be distributive. On the other hand, it was proved by R. Halaš (see [8] and [9]) that if $\mathbf{P}$ is a finite poset and $\operatorname{DM}(\mathbf{P})$ denotes its Dedekind-MacNeille completion of $\mathbf{P}$ (which is a lattice) then $\mathbf{P}$ is distributive if and only if $\operatorname{DM}(\mathbf{P})$ has this property. Unfortunately, we do not know if the Dedekind-MacNeille completion of a uniquely complemented poset is again uniquely complemented. Hence we will use a different method in order to obtain our result.

According to Lemma 3.2, a uniquely complemented poset satisfying LU-identity (2) is a De Morgan poset. A morphism $g$ between De Morgan posets is an order preserving mapping satisfying $g(0)=0, g(1)=1$ and $g\left(x^{\prime}\right)=(g(x))^{\prime}$ for all $x$. A morphism $g$ between De Morgan posets is called order reflecting if $g(x) \leq g(y)$ implies $x \leq y$. We have the following theorem:

Theorem 4.3 If $\mathbf{P}=\left(P, \leq,{ }^{\prime}, 0,1\right)$ is a uniquely complemented atomic poset satisfying $L U$ identity (2) and A denotes the set of its atoms then $\mathbf{P}$ is atomistic, and $f: P \rightarrow 2^{A}$ defined by $f(x):=\{p \in A \mid p \leq x\}$ for all $x \in P$ is an order reflecting morphism of De Morgan posets preserving existing joins and meets.

Proof Let $a, b \in P$ and $p \in A$. Note first that $\mathbf{P}$ is a distributive De Morgan poset. Let us check condition (O4). Clearly, if $a \leq p^{\prime}$ then $p \wedge a=0$. Conversely, if $p \wedge a=0$ then using LU-identity (2) we obtain

$$
a^{\prime} \in U\left(a^{\prime}, 0\right)=U\left(a^{\prime}, L(a, p)\right)=U\left(a^{\prime}, p\right) \subseteq U(p),
$$

i.e., $p \leq a^{\prime}$. Since $\mathbf{P}$ is a De Morgan poset we have $a \leq p^{\prime}$. Let us show that $\mathbf{P}$ is atomistic. Assume $b \in U(f(a))$. Then from LU-identity (2), from $f(a) \subseteq f(b)$ and from condition (O6) we obtain

$$
U\left(a^{\prime}, b\right)=U\left(a^{\prime}, L(a, b)\right) \subseteq U\left(f\left(a^{\prime}\right) \cup f(a)\right)=U(A)=\{1\} .
$$

Hence $U\left(a^{\prime}, b\right)=\{1\}$. According to Theorem 3.5, $\mathbf{P}$ is distributive. Therefore we have

$$
\begin{aligned}
a \in L(a) & =L(a, 1)=L\left(a, U\left(a^{\prime}, b\right)\right)=L\left(U\left(L\left(a, a^{\prime}\right), L(a, b)\right)\right)=L(U(0, L(a, b))) \\
& =L(U(L(a, b)))=L(a, b) \subseteq L(b)
\end{aligned}
$$


Hence $a \leq b$, i.e., $a=\bigvee f(a)$. Since $f(a) \subseteq f(b)$ implies $a=\bigvee f(a) \leq \bigvee f(b)=b$, $f$ is order reflecting.

Now we will assume that the poset considered is even atomistic. Under this relatively strong condition we can prove the following lemma which yields a connection to LU-identities (2) and (3).

Lemma 4.4 Every uniquely complemented atomistic poset $\mathbf{P}=\left(P, \leq,{ }^{\prime}, 0,1\right)$ satisfies condition (O4) and both LU-identities (2) and (3).

Proof Let $A$ denote the set of all atoms of $\mathbf{P}$ and let $a, b \in P$ and $p \in A$. First we check condition (O4). Clearly, if $a \leq p^{\prime}$ then $p \wedge a=0$. Conversely, assume $p \wedge a=0$. Let $q \in A$ with $q \leq a$. Then $q \neq p$. Hence $q \leq p^{\prime}$ according to condition (O3). This yields $a=\bigvee\{x \in A \mid x \leq a\} \leq p^{\prime}$. Now we will check LU-identity (3). Evidently, $L\left(a^{\prime}, b\right) \subseteq L\left(a^{\prime}, U(a, b)\right)$. Conversely, assume $c \in L\left(a^{\prime}, U(a, b)\right)$ and $p \leq c$. Then $p \leq a^{\prime}$ and $p \in L(U(a, b))$. According to condition (O5) we obtain that $p \leq a$ or $p \leq b$. Since $p \leq a^{\prime}$ we have $p \leq b$. Hence $p \in L\left(a^{\prime}, b\right)$, i.e., $c=\bigvee\{x \in A \mid x \leq c\} \in$ $L\left(a^{\prime}, b\right)$. Since LU-identity (3) implies LU-identity (2) according to Lemma 3.4 we are finished.

Although the following theorem was already proved by B. N. Waphare and V. V. Joshi ([15], Theorem 8), it also follows immediately from Lemma 4.4 and Theorem 3.5.

\section{Theorem 4.5 Every uniquely complemented atomistic poset is distributive.}

The previous theorem enables us to formulate a nice result where the strong assumption of atomisticity is replaced by an essential weaker one. For this purpose we need the following definition: An element $a$ of a poset $(P, \leq)$ is called completely join-irreducible if it is not the least element of $(P, \leq)$ and if the following holds: If $a$ is the supremum of a non-empty subset $B$ of $P$ then $a \in B$.

Theorem 4.6 Every uniquely complemented poset $\mathbf{P}=\left(P, \leq,{ }^{\prime}, 0,1\right)$ satisfying the descending chain condition such that its completely join-irreducible elements are atoms is distributive.

Proof Since $\mathbf{P}$ satisfies the descending chain condition it is atomic. From [12], Proposition 2.2, we know that every element of $P$ is a join of completely join-irreducible elements. Since every completely join-irreducible element is an atom we get that $\mathbf{P}$ is atomistic. From Theorem 4.5 we have that $\mathbf{P}$ is distributive.

Applying LU-identity (2), assumptions can be further simplified as the following theorem shows.

Theorem 4.7 Every uniquely complemented poset $\mathbf{P}=\left(P, \leq,{ }^{\prime}, 0,1\right)$ satisfying the descending chain condition and LU-identity (2) is atomistic.

Proof Let $A$ denote the set of all atoms of $\mathbf{P}$. Since $\mathbf{P}$ satisfies the descending chain condition it is atomic. Let $j$ be a completely join-irreducible element of $\mathbf{P}$ and suppose $j \notin A$. Then there exists an atom $p$ of $\mathbf{P}$ with $p<j$. From LU-identity (2) we have

$$
U\left(p, L\left(p^{\prime}, j\right)\right)=U(p, j)=U(j) .
$$


This shows $j=\bigvee\left(L\left(p^{\prime}, j\right) \cup\{p\}\right)$. Since $j$ is a completely join-irreducible and $p<j$, we have $p<j \leq p^{\prime}$, a contradiction. Hence $j \in A$. Using again [12], Proposition 2.2, we know that every element of $P$ is a join of completely join-irreducible elements and hence of atoms.

We conclude our investigations with a theorem connecting modularity and distributivity in uniquely complemented posets satisfying the descending chain condition.

Theorem 4.8 Every uniquely complemented modular poset $\mathbf{P}=\left(P, \leq,{ }^{\prime}, 0,1\right)$ satisfying the descending chain condition is distributive.

Proof Let $A$ denote the set of all atoms of $\mathbf{P}$. Since $\mathbf{P}$ satisfies the descending chain condition it is atomic. Let $j$ be a completely join-irreducible element of $\mathbf{P}$ and suppose $j \notin A$. Then there exists an atom $p$ of $\mathbf{P}$ with $p<j$. Since $\mathbf{P}$ is modular, we have

$$
L\left(U\left(p, L\left(p^{\prime}, j\right)\right)\right)=L\left(U\left(p, p^{\prime}\right), j\right)=L(1, j)=L(j) .
$$

This shows $j=\bigvee\left(L\left(p^{\prime}, j\right) \cup\{p\}\right)$. By the same argument as in the proof of Theorem 4.7 we get a contradiction. Hence $j \in A$. According to Theorem 4.6, $\mathbf{P}$ is distributive.

Acknowledgments Open access funding provided by Austrian Science Fund (FWF).

Open Access This article is distributed under the terms of the Creative Commons Attribution 4.0 International License (http://creativecommons.org/licenses/by/4.0/), which permits unrestricted use, distribution, and reproduction in any medium, provided you give appropriate credit to the original author(s) and the source, provide a link to the Creative Commons license, and indicate if changes were made.

\section{References}

1. Birkhoff, B.G.: Lattice Theory. AMS, Providence, R. I. ISBN 0-8218-1025-1 (1979)

2. Chajda, C.I.: Complemented ordered sets. Arch. Math. (Brno) 28, 25-34 (1992)

3. Chajda, I., Länger, H., Padmanabhan, R.: Single identities forcing lattices to be Boolean. Math. Slovaca (to appear)

4. Chajda, I., Padmanabhan, R.: Lattices with unique complementation. Acta. Sci. Math. (Szeged) 83, 3134 (2017)

5. Chajda, I., Rachunek, J.: Forbidden configurations for distributive and modular ordered sets. Order 5, 407-423 (1989)

6. Dilworth, D.R.P.: Lattices with unique complements. Trans. Amer. Math. Soc. 57, 123-154 (1945)

7. Grätzer, G.G.: Lattice Theory: Foundation. Birkhäuser/Springer, Basel (2011). ISBN 978-3-0348-00174

8. Halas, R.: Annihilators and ideals in distributive and modular ordered sets. Acta Univ. Palack. Olomuc. Fac. Rerum Natur. Math. 34, 31-37 (1995)

9. Halas, R.: Some properties of Boolean ordered sets. Czechoslov. Math. J. 46, 93-98 (1996)

10. Larmerová, J., Rachunek, J.: Translations of distributive and modular ordered sets. Acta Univ. Palack. Olomuc. Fac. Rerum Natur. Math. 27, 13-23 (1988)

11. Niederle, N.J.: Boolean and distribuive ordered sets: Characterization and representation by sets. Order 12, 189-210 (1995)

12. Parameshwara Bhatta, S., Ramananda, H.S.: A note on irreducible elements in a finite poset. Intern. J. Algebra 4, 669-675 (2010)

13. Rachunek, R.J.: Translations des ensembles ordonnés. Math Slovaca 31, 337-340 (1981)

14. Salii, S.V.N.: Uniquely Complemented Lattices. Moskva, Nauka (1984). (in Russian)

15. Waphare, B.N., Joshi, V.V.: On uniquely complemented posets. Order 22, 11-20 (2005) 